\title{
Addressing physical inactivity in Omani adults: perceptions of public health managers
}

\author{
Ruth M Mabry ${ }^{1, *,}$, Zakiya Q Al-Busaidi ${ }^{2}$, Marina M Reeves ${ }^{1}$, Neville Owen ${ }^{1,3}$ and \\ Elizabeth G Eakin ${ }^{\prime}$ \\ ${ }^{1}$ Cancer Prevention Research Centre, School of Population Health, The University of Queensland, Herston, \\ Australia: ${ }^{2}$ Department of Family Medicine and Public Health, College of Medicine, Sultan Qaboos University, \\ Oman: ${ }^{3}$ Behavioural Epidemiology Laboratory, Baker IDI Heart \& Diabetes Institute, Sydney, Australia
}

Submitted 15 June 2012: Final revision received 23 October 2012: Accepted 9 November 2012: First published online 25 January 2013

\begin{abstract}
Objective: To explore barriers and solutions to addressing physical inactivity and prolonged sitting in the adult population of Oman.

Design: Qualitative study involving semi-structured interviews that took place from October 2011 to January 2012. Participants were recruited through purposive sampling. Data collection and analysis was an iterative process; later interviews explored emerging themes. Interviews were audio-recorded and transcribed and continued until data saturation; this occurred by the tenth interviewee. Thematic content analysis was carried out, guided by an ecological model of health behaviour.

Setting: Muscat, Oman.

Subjects: Ten mid-level public health managers.

Results: Barriers for physical inactivity were grouped around four themes: (i) intrapersonal (lack of motivation, awareness and time); (ii) social (norms restricting women's participation in outdoor activity, low value of physical activity); (iii) environment (lack of places to be active, weather); and (iv) policy (ineffective health communication, limited resources). Solutions focused on culturally sensitive interventions at the environment (building sidewalks and exercise facilities) and policy levels (strengthening existing interventions and coordinating actions with relevant sectors). Participants' responses regarding sitting time were similar to, but much more limited than those related to physical inactivity, except for community participation and voluntarism, which were given greater emphasis as possible solutions to reduce sitting time.

Conclusions: Given the increasing prevalence of chronic disease in Oman and the Arabian Gulf, urgent action is required to implement gender-relevant public health policies and programmes to address physical inactivity, a key modifiable risk factor. Additionally, research on the determinants of physical inactivity and prolonged sitting time is required to guide policy makers.
\end{abstract}

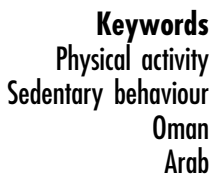

Participation in physical activity extends life expectancy, reduces premature mortality related to CVD, diabetes and cancer, and promotes mental health ${ }^{(1,2)}$. Sedentary behaviour (any waking behaviour involving very low energy expenditure while sitting or reclining ${ }^{(3)}$ ) is a newly identified risk for diabetes, obesity and biomarkers of chronic disease risk like abnormal glucose metabolism and dyslipidaemia $^{(4,5)}$. Recent extensive secondary analyses of an Omani population-based survey established the association of physical inactivity and sitting time with the metabolic syndrome and identified population subgroups

$\dagger$ Address for correspondence: PO Box 476, Al Atheiba, Postal Code 130, Muscat, Oman. who were the most inactive and who sat the longest ${ }^{(6,7)}$. Promoting physical activity and reducing prolonged sitting have the potential to be key strategies that could address the high prevalence of type 2 diabetes, CVD and other chronic diseases in Oman and other countries of the Gulf Cooperation Council (GCC; Bahrain, Kuwait, Oman, Qatar, Saudi Arabia, United Arab Emirates) ${ }^{(8-10)}$.

Ecological models of physical activity and sedentary behaviour identify multilevel influences including intrapersonal, social, physical environment and public policy ${ }^{(11-13)}$. Key public health documents addressing physical activity - particularly the Centers for Disease Control and Prevention's Guide to Strategies for Increasing Physical Activity in the Community ${ }^{(14)}$, the WHO guide to 
increasing physical activity ${ }^{(15)}$ and The Toronto Charter for Physical Activity: A Global Call for Action ${ }^{(16)}$ - identify the importance of such broadly based perspectives that can guide environmental and policy initiatives.

Public health professionals can have an influential role in advocating and shaping evidence-based health policy and programmes ${ }^{(17)}$. However, in the GCC, where limited evidence is available on the determinants of physical inactivity and prolonged sitting ${ }^{(18-20)}$, an understanding of public health professionals' perceptions of the influences on these health risk behaviours is required in order to inform relevant public health initiatives. The present qualitative study, guided by an ecological model of physical activity and sedentary behaviour ${ }^{(11,13)}$, aimed to identify policy and programme options to address physical inactivity and prolonged sitting in the adult population of Oman.

\section{Methods}

As part of a series of studies on physical inactivity and sitting time in $\operatorname{Oman}^{(6,7)}$, the current paper presents the results of a semi-structured interview survey, guided by an ecological model of health behaviour, undertaken to explore public health managers' perceptions about addressing these health risk behaviours in Oman. Since prolonged sitting is an emerging risk factor about which little is known in the GCC, this issue was included in the study to examine if it was viewed as a risk factor within the broader context of addressing physical inactivity, and whether barriers and solutions would differ from those put forward for physical inactivity. The study was approved by the Research and Ethical Review Committee, Ministry of Health, Oman and the Behavioural and Social Sciences Ethical Review Committee, the University of Queensland, Australia.

\section{Data collection}

Semi-structured interviews were conducted by R.M.M. between the months of October 2011 and January 2012 with ten mid-level national public health managers in the Ministry of Health, Oman recruited by purposive sampling ${ }^{(21)}$. An Information Note describing the study and summarizing the available evidence from Oman ${ }^{(6,7)}$ was shared with the participants as part of their invitation letter. Follow-up telephone calls were made to arrange a time and place for the interview. Although Arabic was the mother tongue for all participants, all interviews were conducted in English, since it is the common working language in the health sector in Oman.

The interview guide contained open-ended questions eliciting participants' feedback on the barriers to reducing physical inactivity and prolonged sitting, and their suggestions on possible programmes and policies to address these behaviours (see Table 1). Interviews began with a brief overview of the study. Participants' verbal consent to be interviewed and to be audio-recorded was obtained. Each participant described his/her role within the Ministry of Health. Participants' perceptions of barriers and possible solutions were explored based on the evidence generated from a series of studies of these behaviours in Oman $^{(6,7)}$. The duration of interviews ranged from 31 to $69 \mathrm{~min}$ (average time: $50 \mathrm{~min}$ ). Interviews were digitally audio-recorded, transcribed verbatim and numbered to ensure anonymity of participants' responses. Once initial transcripts were drafted, R.M.M. compared them with the original recordings and hand-written notes to ensure accurate transcription.

\section{Data analysis}

Data analysis followed a hybrid of thematic content analysis and a framework approach ${ }^{(22,23)}$ in line with the key aims of the study (identifying the barriers and solutions to physical inactivity and sedentary behaviour in Oman) and from the perspective of an ecological approach to understanding the determinants of health behaviours. Initial transcripts were read several times by authors R.M.M. and Z.Q.A. followed by open coding, grouping and categorizing data according to emerging themes. A coding scheme was then developed based on the major recurrent themes. All transcripts were then reviewed and segments of text coded by R.M.M. (and a sub-sample by Z.Q.A.) based on the coding scheme. Where differing interpretations of themes occurred, transcripts were re-reviewed until consensus was reached. Although an

\section{Table 1 Interview schedule}

1. I know we have worked together for a long time, but to ensure consistency in all my interviews, can you tell me how long you have worked in public health and describe for me the current work you do for the Ministry of Health?

2. How does your work involve addressing physical activity?

3. How important do you think addressing physical activity is among the various public health priorities for the Ministry of Health?

Share the evidence of the association of physical activity on the metabolic syndrome and the correlates of physical activity in Oman.

4. Do you think these results from Sur are similar to what we would see from the rest of Oman? If no, what is different about Sur? If yes: why?

5. If we think about the groups identified as being at the highest risk of physical inactivity, what are the key barriers to doing physical activity for these groups?

6. Based on these reflections, what suggestions do you have on introducing new programmes and policies that address physical inactivity in Oman?

Share two slides on the evidence of the association of sitting time on the metabolic syndrome and the correlates of sitting time in Oman.

7. How important a priority do you think addressing sitting time is among the various public health priorities for the Ministry of Health?

8. If we think about the groups identified as being at the highest risk of extended sitting time, what are some barriers to reducing sitting time?

9. What suggestions do you have about possible programmes and policies to address these groups?

10. Is there anything else you would like to add? 
ecological model was used as a guide, the researchers followed the themes that emerged from the data.

Data collection and analysis was an iterative process, whereby later interviews allowed the exploration of themes and perceptions generated from early interviews as well as the generation of new themes. Interviews continued until data saturation, when interviews no longer brought new themes ${ }^{(21,24)}$. To enhance rigour, analyses included searching for negative cases in code and theme development ${ }^{(24,25)}$. Analysis was done manually rather than using qualitative analysis software, since the sample size made this feasible. The common themes are presented in the Results section according to level of influence (intrapersonal, social, physical environmental and public policy) identified by ecological models ${ }^{(11,13)}$ and using extracts of participants' responses as headings and as primary data.

\section{Results}

A total of ten interviews were conducted. All participants had a postgraduate degree in a field related to public health (including six with a medical background) and extensive public health experience (mean years in public health: 15 years). Except for one person, participants were Omani; half were women.

The participants identified numerous barriers to physical activity across the four main levels of influence described in ecological models (intrapersonal, social, physical environment and public policy) and proposed a wide range of public health solutions. Table 2 provides a summary of the emergent themes and sub-themes; sub-themes are ordered according to how often they were mentioned by participants. Although the findings presented below and in Table 2 are according to discrete themes, many participants' responses were multi-factorial; for example, combining the social and physical environment (i.e. the cultural perception that sports clubs are for young people) or intrapersonal and social influences (i.e. not having time because of family and social responsibilities).

\section{Perceptions about physical inactivity}

Intrapersonal - 'They have hundred ... excuse[s]'

The most common personal-level barrier to Omanis engaging in physical activity mentioned by the interview participants was lack of motivation. In addition, several participants mentioned lack of awareness of the importance of physical activity and lack of time due to conflicting work and family responsibilities:

'I mean, you look at the people you know before they get married and after they get married, even men, you become tied up with social barriers, you get tied up with the social engagement of your family to the extent that you cannot have control over your own life.' (Participant 2)
Social - 'It's a stigma if you run'

One of the most frequently mentioned barriers from the interviews was the socio-cultural norms that restrict women's participation in outdoor physical activity, including their conservative dress:

'In our culture, if I was a woman interested in walking, and I walk by myself in the middle of the street, you know, it doesn't look good. You know, it looks bad. Still people will look at it in a negative way.' (Participant 1)

A few participants mentioned social restrictions due to a person's age, particularly for women; however, one person noted that older people may be more active than their younger counterparts.

The other common barrier mentioned was the low value placed on physical activity in Oman:

'The definition of physical activity is incorrect. In the West, physical activity is seen as an important part of life, like prayer and going to the mosque. But not so in Oman. It is not seen as important.' (Participant 4)

Other issues mentioned by the participants were linked to modernization and urbanization, such as the cultural norm of employing domestic workers, using private vehicles rather than public transport which is known to promote physical activity ${ }^{(2)}$, and the limited availability, and a culturally negative perception of, public transportation:

'.... and the lack of [public] transportation, and the taboo associated. If you use a bus, everybody will question you. It is not considered normal.' (Participant 2)

\section{Physical environment - 'Where will you go?'}

The physical environment was the most frequently mentioned barrier for physical activity. Several participants spoke more generally about the limited availability of places to be physically active or about the inadequate built environment for physical activity:

'Walking, you have to have a pleasant place. You can't walk everywhere. And you know that, if there's no pavement, a lot of cars, it's not a place for walking.' (Participant 9)

Several participants also talked about the inaccessibility of government sports clubs due to distance and lack of transportation and their limited focus, and of private exercise facilities because of their cost or low quality:

'Y'know, I feel the facilities are not well equipped, I mean, [particularly] for females... If it is there, it is not fully equipped. For example, the sports clubs in the wilayats [districts], it is only meant for males. Yes, we saw in the news that females are welcome to go there but I don't think ... it is easy.' (Participant 10)

Several participants mentioned the hot desert climate as a barrier to being physically active; however, one 
Table 2 Public health managers' perceptions on barriers and solutions to address physical inactivity and prolonged sitting in Oman

\begin{tabular}{|c|c|}
\hline Theme & Sub-theme \\
\hline \multicolumn{2}{|l|}{ Barriers to addressing physical inactivity } \\
\hline \multirow[t]{3}{*}{ Intrapersonal } & Lack of motivation \\
\hline & Lack of awareness \\
\hline & Lack of time \\
\hline \multirow[t]{6}{*}{ Social environment } & Social restrictions on women \\
\hline & Low value placed on physical activity \\
\hline & Dependence on motorized transport \\
\hline & Social restrictions for older people \\
\hline & Cultural norm to employ domestic workers \\
\hline & Negative association of public transport/taxis \\
\hline \multirow[t]{6}{*}{ Physical environment } & Inadequate infrastructure \\
\hline & Limited access to places to be active \\
\hline & Limited access to government sports clubs \\
\hline & Limited access to private sports clubs \\
\hline & Weather \\
\hline & Sedentary work setting \\
\hline \multirow[t]{4}{*}{ Public policy environment } & Ineffective health communication \\
\hline & Limited resources \\
\hline & Focus on curative \\
\hline & Lack of healthy public policies \\
\hline \multicolumn{2}{|l|}{ Solutions to address physical activity } \\
\hline \multirow[t]{2}{*}{ Build conducive environment } & Build supportive infrastructure \\
\hline & Build culturally appropriate infrastructure \\
\hline Community participation & Identify and work with relevant groups and organizations \\
\hline \multirow[t]{3}{*}{ Multisectoral coordination } & Work with other sectors \\
\hline & Advocate for supportive environment \\
\hline & Establish a national health promotion body \\
\hline \multirow[t]{9}{*}{ Strengthen Ministry of Health policies and programmes } & Improve health communication \\
\hline & Use settings approach \\
\hline & Conduct research \\
\hline & Promote physical activity for staff \\
\hline & Review human resource requirements \\
\hline & Strengthen primary health care \\
\hline & Region-specific action \\
\hline & Establish unit within the Ministry of Health \\
\hline & Address physical activity and diet together \\
\hline \multicolumn{2}{|l|}{ Barriers to addressing prolonged sitting } \\
\hline \multirow{2}{*}{ Intrapersonal } & Lack of awareness \\
\hline & Lack of motivation \\
\hline \multirow[t]{3}{*}{ Social environment } & Sedentary culture \\
\hline & Limited culturally appropriate activities for women \\
\hline & Cultural norm to employ domestic workers \\
\hline Physical environment & Limited access to non-sedentary activities \\
\hline & Sedentary work setting \\
\hline \multicolumn{2}{|l|}{ Solutions to address prolonged sitting } \\
\hline Community participation & Identify and work with relevant groups and organizations \\
\hline Multisectoral coordination & Advocate for supportive environment for physical activity \\
\hline & Work with other sectors \\
\hline \multirow[t]{4}{*}{ Strengthen Ministry of Health policies and programmes } & Raise awareness \\
\hline & Use settings approach \\
\hline & Address physical activity and sitting time together \\
\hline & Conduct research \\
\hline
\end{tabular}

noted that in some areas, the relatively cold winter months may prevent some people from being active.

Public policy - 'Our programme mostly just concentrate[s] on awareness'

The concerns with public policy were largely related to current interventions by the Ministry of Health. Many participants spoke of the inadequacy of health communication (including both mass media and printed educational materials):

'As you know, we are delivering health messages in terms of TV programmes or in terms of leaflet, posters... Is this the correct kind of way to reach those people who are the new generation, who know about the internet, who have other methods, attractive methods, to reach them? So, we need to change the health message delivery. We need to study this group ... how they can digest the health message.' (Participant 6)

Several also noted the limited resources available at both the national and institutional level to address physical inactivity:

'But most of the programme[s] in the government are not set up to do this type of intervention 
because we don't have the staff, the human resources to follow-up, to implement these things. That's why we only do awareness. It's the easy way because that's the only way we can educate people. But to change behaviour, you really need to build interventions that we really support people ... to continue.' (Participant 1)

Also, two participants mentioned that the focus in the Ministry of Health is on curative medicine rather than prevention, and that health is not considered in all government policies, due to the limited engagement by non-health stakeholders in public health issues.

\section{Participants' solutions to address physical inactivity}

Participants provided a wide range of solutions to address physical inactivity, which can be grouped into two main areas: (i) building a supportive physical environment and (ii) building a supportive policy environment within the health sector as well as within other sectors.

\section{Supportive physical environment - 'We need ... good pavements'}

Many participants stressed the importance of having a supportive physical infrastructure (including transport to available sports facilities) to encourage people to be active:

'If we encourage walking to schools, okay ... this, of course, means to have good infrastructure, places, streets, or places which you should be safe because you're expecting the child may cross the road. For example, there should be facilities to make this easier... So it needs also, some ... improvement in the infrastructure, streets.' (Participant 3)

Several participants emphasized that the infrastructure should be culturally appropriate such as having separate spaces for women. In fact, one participant, in advocating for gender-segregated spaces, reported of communities in Oman that built gender-segregated walkways:

'As I told you, in the beginning we thought this [segregated] walking pathway nobody would utilize. But I was surprised and amazed. So, maybe when we make things available for the people, [they] will go for it.' (Participant 8)

\section{Supportive bealth policy environment - 'You really need to build interventions'}

The participants had numerous policy solutions, most of them related to the Ministry of Health. The most commonly mentioned issue was the need to strengthen health communication:

'We should have ... some type of repeated campaigns on this just to increase the awareness. For example, it will happen here in Muscat but when you go into the regions it will rarely happen, these campaigns. It is just ... haphazard activities.' (Participant 5)

However, two participants felt that it would be better to focus on policy to support active living, such as development and transport policies that would make regular walking the easy choice.

Many participants also suggested strengthening the school health programme and conducting research to evaluate current interventions. Although only one participant mentioned using the workplace as a setting to promote physical activity, several focused specifically on how the Ministry of Health should promote physical activity among its own staff:

'Actually before just going to anybody and asking them to do physical activity to ... follow the diet or whatever, we need to start initially from our house. We have a lot of people employed in the Ministry of Health. Now, 27000 people are now employed in the Ministry of Health.' (Participant 6)

In order for the Ministry of Health to address physical inactivity, several participants emphasized the need for more staff with related experience in physical activity and for improving primary prevention within health-care settings:

We need to have a very well-established well clinic ... Now we have this national screening programme for above 40. But we need to have a well clinic starting from 20. We need to go just to the health centres to check annually... We don't have this service. We don't have this person. We have health educators; we have dietitians. But their capacities really to that extent to direct people ... they need to be monitored.' (Participant 8)

\section{Coordinate with other stakebolders - 'We need to have other partners'}

Most participants highlighted the importance of working with other sectors and suggested that the Ministry of Health does more advocacy in order to gain political support and engagement from key stakeholders:

'I think what the Ministry of Health should demonstrate ... the trend in chronic diseases, how this could affect the population and should demonstrate it to all the State Council [upper house of parliament, membership is by appointment], the Shura Council [lower house of parliament, membership is by election], and to the Cabinet.' (Participant 7)

Half of the participants advocated the establishment of a high-level national body on health promotion:

'We should have national health promotion committee or council, let us say, or even organization or ... 
health promotion body that can gather all the sectors together to work in this issue. Ministry of Health cannot do it alone.' (Participant 5)

\section{Perceptions about prolonged sitting - 'We're a culture that likes to sit'}

The participants' responses regarding prolonged sitting were limited compared with those regarding physical inactivity. The key influences on prolonged sitting identified by the participants can be grouped into three main levels consistent with ecological models: (i) intrapersonal (limited awareness of the adverse effects of prolonged sitting); (ii) the social environment (sedentary culture); and (iii) the physical environment (limited places for active leisure). As one participant noted:

'Not most of them they like to go to the beach. And if they go to the beach, BBQ and sitting. Okay? We have few malls, and again, the kids are playing and parents sitting. Know what I mean? So we have limited facilities to go outside.' (Participant 8)

Several participants noted that women, in particular, had limited culturally appropriate options available besides staying at home:

'The ladies ... which have no job, what [option] will they have except sitting at home? ... They cannot just go around roaming between the houses ... socially it's not acceptable.' (Participant 5)

Solutions to address prolonged sitting were similar to those regarding physical inactivity, such as coordinating with other sectors and doing more in school and workplace settings. The most common solution for the Ministry of Health focused largely on raising awareness. Although community participation was suggested by some of the participants as a way to address physical inactivity, community-based activity and voluntarism was mentioned more often as a way to reduce prolonged sitting at home, the alternative choice, particularly for women:

'They can participate in schools in their own community. They can participate to support in the health-care facilities ... on a voluntary basis. We don't encourage people to participate and be part of their society and I think that's why people are sitting at home.' (Participant 1)

\section{Discussion}

The perceptions of public health managers about physical inactivity is an important influence on whether or not available evidence on the health impact of this health risk behaviour will be translated into relevant policy and practice $^{(17)}$. The responses from the current qualitative study reflect perceptions of the multidimensional influences on this behaviour and the multilevel public health solutions that will be needed to address it, consistent with ecological models of health behaviour ${ }^{(11,12)}$. Given that sedentary behaviour is a new area of research ${ }^{(4)}$, the more limited discussion on prolonged sitting time is not surprising.

The socio-cultural barriers observed by the participants, particularly the restrictions for women, are seen in other countries within the $\operatorname{GCC}^{(18-20)}$. For example, a recent qualitative study from the United Arab Emirates reported that women do not have space at home for indoor exercise and families do not allow them to go for a walk alone ${ }^{(18)}$. Similar barriers have been reported from other Arab communities in the Middle East ${ }^{(27,28)}$ and the $\mathrm{USA}^{(29,30)}$. The social norms that limit women's involvement in physical activity may be an important contributor to the prevalence rates of overweight/obesity and the metabolic syndrome, which are much higher for women than for men in the GCC region ${ }^{(8,9)}$.

Several of the other barriers mentioned by the participants (i.e. dependence on motorized transportation, sedentary work settings, dependence on domestic workers) reflect the socio-economic transition in the country. Evidence indicates that in many parts of the world, this transition is resulting in higher intakes of meats, fats and total energy, and increased physical inactivity, particularly in the occupational and transport domains, that, in turn, is increasing the prevalence of chronic diseases ${ }^{(31-33)}$. Limited evidence is available on the trends in physical inactivity prevalence in countries of the GCC. However, given the other available evidence from the GCC region, such as the increasing consumption of fats and total energy ${ }^{(34)}$ and the increasing prevalence of overweight, obesity, hypertension and type 2 diabetes $^{(8,35)}$, it is likely that the prevalence of physical inactivity is also increasing.

Recommendations by participants on how to address physical inactivity focused largely on population-based interventions such as mass media health communications and building appropriate physical infrastructure to support physical activity. These recommendations are in line with The Toronto Charter for Physical Activity ${ }^{(16)}$ and the supporting document on the seven best investments for physical activity ${ }^{(26)}$. Many such interventions, as clearly articulated by the participants, require intersectoral engagement. However, a whole-of-government approach in promoting health has not yet taken root in the region ${ }^{(18,35)}$. Since ministries of health bear the cost of managing chronic disease in the GCC, the onus is on the health sector to advocate for multisectoral interventions. These interventions can be based on available evidence ${ }^{(36,37)}$, global public health guidance ${ }^{(16,26,38)}$ and experience from within the GCC region, such as the Kuwait National Physical Activity $\operatorname{Plan}^{(39)}$ and the development of culturally appropriate built environment (i.e. gender-segregated walkways and exercise facilities) in some communities in $\operatorname{Oman}^{(40,41)}$.

Individual-level interventions, such as incorporating physical activity screening and patient education for 
chronic disease prevention in primary health-care services $^{(26)}$, were mentioned by only a few participants, despite the Ministry of Health being the main provider of health-care services in the country. Researchers have begun to test such individual-level interventions, adapted for the Arab context, with some success ${ }^{(30,42-44)}$. However, given the sparse evidence available, further research on appropriate interventions for Arab populations is urgently needed.

The more limited discussion on prolonged sitting, including the limited number of suggested interventions, is an indication that sedentary behaviour as an independent health risk factor is a relatively new concept - not only in Oman but also globally ${ }^{(4)}$. Only seven countries have guidelines, most of which target children and focus on limiting television viewing time and other screen time ${ }^{(45)}$. Raising awareness in concert with continued behavioural epidemiology research is required. Making use of the growing body of evidence on prolonged sitting ${ }^{(13)}$ can guide the research agenda appropriate for the region.

The main limitation of the present study is the restriction of the sample to mid-level public health programme managers. Obtaining their views is important in the context of public health planning and was the main purpose of the study; however, they are not representative of all health professionals working in the Ministry of Health, Oman or of society at large. The interviewer, author R.M.M., has worked with all study participants for several years, which facilitated the arrangement of interviews; however, familiarity may also have led to bias, with participants providing socially desirable responses. All participants have a very good working knowledge of English, but their responses may have been limited since the interviews were not conducted in their mother tongue, Arabic.

Study findings are important given the increasing prevalence of chronic disease and the limited evidence available regarding physical inactivity in Oman and the GCC. Advocacy related to study implications has already been initiated through formal presentations of the findings at the Ministry of Health and the College of Medicine, Sultan Qaboos University by the lead author. Opportunities for advocacy through national seminars, meetings with key stakeholders and informal discussion groups are also emerging.

\section{Conclusion}

As the economies of countries of the GCC, including Oman, continue to grow, efforts are needed to offset the negative health impacts of socio-economic development. Urgent multilevel action to reduce physical inactivity and prolonged sitting is required and should be sensitive to the socio-cultural context of the population of the GCC and well as the physical and built environment, particularly the harsh weather conditions. Community-based interventions addressing physical inactivity should be expanded, based on locally successful models ${ }^{(40)}$. Given the high prevalence of chronic disease in the region, significant behavioural epidemiology research on physical inactivity and prolonged sitting is required to fill the knowledge gap and guide policy makers.

\section{Acknowledgements}

Sources of funding: M.M.R., N.O. and E.G.E. are supported by Programme and Fellowship Grants from the National Health and Medical Research Council of Australia and by Research Infrastructure funding from Queensland Health. N.O. is also supported by Research Infrastructure funding from the Victorian Government. Conflicts of interest: The authors declare to have no conflicts of interest. Authors' contributions: R.M.M. was responsible for the initial research concept, preparation of the study questionnaire, data collection, data analysis and interpretation, preparation of the manuscript and tables, and revision of the paper. Z.Q.A. contributed to the study design, assisted with data collection, analysis and interpretation, helped in preparation of the tables and assisted in manuscript editing. M.M.R. contributed to the initial concept and study design, data interpretation and manuscript editing. N.O. assisted with the initial concept and study design and manuscript editing. E.G.E. supervised design and implementation of the study and assisted with the manuscript writing and editing. Acknowledgements: The authors wish to thank the participants for sharing their thoughts and for the pleasant, honest and often humorous interactions.

\section{References}

1. Haskell WL, Lee IM, Pate RR et al. (2007) Physical activity and public health: updated recommendation for adults from the American College of Sports Medicine and the American Heart Association. Circulation 116, 1081-1093.

2. World Health Organization (2002) Reducing Risks, Promoting Healthy Life. World Health Report 2002. Geneva: WHO.

3. Sedentary Behaviour Research Network (2012) Standardized use of the terms 'sedentary' and 'sedentary behaviours'. Appl Physiol Nutr Metab 37, 540-542.

4. Owen N, Healy GN, Matthews CE et al. (2010) Too much sitting: the population health science of sedentary behavior. Exerc Sport Sci Rev 38, 105-113.

5. Thorp AA, Owen N, Neuhaus M et al. (2011) Sedentary behaviors and subsequent health outcomes in adults a systematic review of longitudinal studies, 1996-2011. Am J Prev Med 41, 207-215.

6. Mabry R, Winkler E, Eakin E et al. (2012) Correlates of Omani adults' physical inactivity and sitting time. Public Health Nutr (Epublication ahead of print version).

7. Mabry RM, Winkler EA, Reeves MM et al. (2012) Associations of physical activity and sitting time with the metabolic syndrome among Omani adults. Obesity (Silver Spring) 20, 2290-2295.

8. Ng SW, Zaghloul S, Ali HI et al. (2011) The prevalence and trends of overweight, obesity and nutrition-related non-communicable diseases in the Arabian Gulf States. Obes Rev 12, 1-13. 
9. Mabry R, Reeves M, Eakin E et al. (2010) Gender differences in prevalence of the metabolic syndrome in Gulf Cooperation Council countries: a review. Diabet Med 27, 593-597.

10. International Diabetes Federation (2011) IDF Diabetes Atlas, 5th ed. Brussels: IDF.

11. Sallis JF, Owen N \& Fisher EB (2008) Ecological models of health behavior. In Health Behavior and Health Education: Theory, Research, and Practice, 4th ed., pp. 465-482 [K Glanz, K Rimer and K Viswanath, editors]. San Francisco, CA: Jossey-Bass

12. King AC, Stokols D, Talen E et al. (2002) Theoretical approaches to the promotion of physical activity: forging a transdisciplinary paradigm. Am J Prev Med 23, 15-25.

13. Owen N, Sugiyama T, Eakin EE et al. (2011) Adults' sedentary behavior determinants and interventions. $\mathrm{Am} \mathrm{J}$ Prev Med 41, 189-196.

14. US Department of Health and Human Services (2010) The CDC Guide to Strategies for Increasing Physical Activity in the Community. Atlanta, GA: US DHHS.

15. World Health Organization (2007) A Guide for PopulationBased Approaches to Increasing Levels of Physical Activity. Implementation of the WHO Global Strategy on Diet, Physical Activity and Health. Geneva: WHO.

16. Global Advocacy Council for Physical Activity, International Society for Physical Activity and Health (2010) The Toronto Charter for Physical Activity: A Call to Action. http://www. globalpa.org.uk/pdf/totrontocharter-eng-20may2010.pdf

17. Allender S, Gleeson E, Crammond B et al. (2012) Policy change to create supportive environments for physical activity and healthy eating: which options are the most realistic for local government? Health Promot Int 27, 261-274.

18. Ali HI, Baynouna LM \& Bernsen RM (2010) Barriers and facilitators of weight management: perspectives of Arab women at risk for type 2 diabetes. Health Soc Care Community 18, 219-228.

19. Berger G \& Peerson A (2009) Giving young Emirati women a voice: participatory action research on physical activity. Health Place 15, 117-124.

20. Amin TT, Suleman W, Ali A et al. (2011) Pattern, prevalence, and perceived personal barriers toward physical activity among adult Saudis in Al-Hassa, KSA. J Phys Act Health 8, 775-784.

21. Dicicco-Bloom B \& Crabtree BF (2006) The qualitative research interview. Med Educ 40, 314-321.

22. Bradley EH, Curry LA \& Devers KJ (2007) Qualitative data analysis for health services research: developing taxonomy, themes, and theory. Health Serv Res 42, 1758-1772.

23. Pope C, Ziebland S \& Mays N (2000) Qualitative research in health care. Analysing qualitative data. BMJ 320, 114-116.

24. Mays N \& Pope C (2000) Qualitative research in health care. Assessing quality in qualitative research. BMJ 320, 50-52.

25. Mays N \& Pope C (1995) Rigour and qualitative research. BMJ 311, 109-112.

26. Global Advocacy for Physical Activity, International Society for Physical Activity and Health (2011) Non Communicable Disease Prevention: Investments that Work for Physical Activity. A complementary document to The Toronto Charter for Physical Activity: A Call to Action. http://www. globalpa.ork.uk/pdf/investments-work.pdf
27. Daoud N (2008) Challenges facing minority women in achieving good health: voices of Arab women in Israel. Women Health 48, 145-166.

28. Shuval K, Weissblueth E, Araida A et al. (2008) The role of culture, environment, and religion in the promotion of physical activity among Arab Israelis. Prev Chronic Dis 5, A88.

29. Kahan D (2011) Arab American college students' physical activity and body composition: reconciling Middle East-West differences using the socioecological model. Res $Q$ Exerc Sport 82, 118-128.

30. Jaber LA, Pinelli NR, Brown MB et al. (2010) Feasibility of group lifestyle intervention for diabetes prevention in Arab Americans. Diabetes Res Clin Pract 91, 307-315.

31. Siegel K, Narayan KM \& Kinra S (2008) Finding a policy solution to India's diabetes epidemic. Health Aff (Millwood) 27, 1077-1090.

32. Popkin BM, Adair LS \& Ng SW (2012) Global nutrition transition and the pandemic of obesity in developing countries. Nutr Rev 70, 3-21.

33. Monda KL, Gordon-Larsen P, Stevens J et al. (2007) China's transition: the effect of rapid urbanization on adult occupational physical activity. Soc Sci Med 64, 858-870.

34. Musaiger AO (2004) Overweight and obesity in the Eastern Mediterranean Region: can we control it? East Mediterr Health J 10, 789-793.

35. Al-Lawati JA, Mabry R \& Mohammed AJ (2008) Addressing the threat of chronic diseases in Oman. Prev Chronic Dis 5, A99.

36. Kahn EB, Ramsey LT, Brownson RC et al. (2002) The effectiveness of interventions to increase physical activity. A systematic review. Am J Prev Med 22, 73-107.

37. Heath GW, Brownson RC, Kruger J et al. (2006) The effectiveness of urban design and land use and transport policies and practices to increase physical activity: a systematic review. J Phys Act Health 3, Suppl. 1, S55-S76.

38. World Health Organization (2004) Global Strategy on Diet, Physical Activity and Health. Geneva: WHO.

39. Ramadan J, Vuori I, Lankenau B et al. (2010) Developing a national physical activity plan: the Kuwait example. Glob Health Promot 17, 52-57.

40. Ministry of Health Oman (2012) Nizwa Healthy Lifestyle Evaluation Report. Nizwa: Directorate General of Health Services, Ministry of Health.

41. Al-Siyabi H (2012) Community participation in health. Presented at CBI Review Forum held in Muscat on 13 February 2012. Muscat: Department of Community-based Initiatives, Ministry of Health.

42. Abduelkarem A \& Sackville M (2009) Changes of some health indicators in patients with type 2 diabetes: a prospective study in three community pharmacies in Sharjah, United Arab Emirates. Libyan J Med 4, 31-36.

43. Kalter-Leibovici O, Younis-Zeidan N, Atamna A et al. (2010) Lifestyle intervention in obese Arab women: a randomized controlled trial. Arch Intern Med 170, 970-976.

44. Midhet FM \& Sharaf FK (2011) Impact of health education on lifestyles in central Saudi Arabia. Saudi Med J32, 71-76.

45. Department of Health \& Department for Children, Schools and Families (2010) Sedentary Behaviour and Obesity: Review of the Current Scientific Evidence. London: Department of Health. 